19 Revue d'histoire du XIXe siècle

Société d'histoire de la révolution de 1848 et des

révolutions du XIXe siècle

$20 / 21 \mid 2000$

Varia

\title{
Pierre-Yves KIRSCHLEGER, La religion de Guizot, Genève, Labor et Fides, 1999, 269 p.
}

Jean-François Jacouty

\section{OpenEdition}

Journals

Édition électronique

URL : http://journals.openedition.org/rh19/228

DOI : 10.4000/rh19.228

ISSN : $1777-5329$

Éditeur

La Société de 1848

Édition imprimée

Date de publication : 1 juin 2000

ISSN : 1265-1354

Référence électronique

Jean-François Jacouty, « Pierre-Yves KIRSCHLEGER, La religion de Guizot, Genève, Labor et Fides, 1999, 269 p. », Revue d'histoire du XIXe siècle [En ligne], 20/21 | 2000, mis en ligne le 04 septembre 2008, consulté le 22 septembre 2020. URL : http://journals.openedition.org/rh19/228 ; DOI : https:// doi.org/10.4000/rh19.228

Ce document a été généré automatiquement le 22 septembre 2020.

Tous droits réservés 


\title{
Pierre-Yves KIRSCHLEGER, La religion de Guizot, Genève, Labor et Fides, 1999, 269 p.
}

\author{
Jean-François Jacouty
}

1 Depuis plusieurs années Guizot, qui était tombé dans l'oubli à la fin de son siècle, est l'objet d'une redécouverte salutaire. Mais les différents aspects de sa personnalité et de son activité restent - encore - inégalement connus. Ainsi le politique a-t-il été plus étudié que l'historien et le chrétien. Comblant une lacune, P.Y. Kirschleger nous apporte une utile contribution à la connaissance de La religion de Guizot. Édition d'un mémoire de maîtrise, cet ouvrage, en fait, couvre un champ plus large que son titre. Démarche fructueuse car, déclare l'auteur dans son introduction, " la religion concerne tout l'homme dans toutes ses dimensions et ne saurait être réduite à une partie de son existence" (p.12). Mettant le chrétien en relation avec l'historien et le politique, P.Y. Kirschleger cherche, d'abord, à " comprendre et faire comprendre l'intrigue de cette vie " (p. 13). Mais tout en refusant d'en dresser " le récit ", il suit un plan - grosso modo - chronologique en trois parties.

La première - une jeunesse protestante au début du XIXe siècle - nous rappelle les éléments constitutifs de la religion de Guizot. C'est, d'abord, l'héritage protestant du Languedoc, renforcé par la " résistance " du Désert à laquelle il a été sensible. Sa mère, qui vivra longtemps à ses côtés, maintiendra la vive mémoire d'un passé autant douloureux qu'héroïque. De cet héritage, et des violences de la Révolution qui ont durement frappé sa famille, Guizot retirera un fort pessimisme. Mais aussi un moralisme rigide, accompagné de ce sentiment d'élection et d'une volonté d'élévation, propres aux minorités aux convictions têtues. Puis sa mère le conduit à Genève pour assurer sa formation intellectuelle, mais sans cesser d'exercer son magistère moral. L'auteur nous décrit l'état de la ville du Refuge: restée d'apparence calviniste, elle professe une religion surtout morale, mais celle-ci, en ce temps d'occupation française, fonde aussi un esprit de liberté et un vrai patriotisme (qui auraient pu être soulignés). Autre forme de "résistance" qui a marqué Guizot. Il reste, pourtant, que le protestantisme genevois ne saurait être assimilé à " la religion naturelle ", comme 
l'affirme l'auteur (p. 34). À preuve la vigoureuse riposte des pasteurs à l'article Genève de l'Encyclopédie : n'accusent-ils pas d'Alembert d'avoir réduit leur religion à un simple déisme! Même s'il est vrai que le " supranaturalisme ", la théologie locale, limite le champ de la foi au profit de la raison, réduisant même la religion à un ethos, l'équilibre entre les deux termes varie néanmoins. Il évolue entre des survivances d'orthodoxie (dont le pasteur Peschier, qui est cité, est un exemple) et les limites de la religion naturelle (illustrée par Jean-Jacques. Rousseau, autre Genevois). Surtout, il aurait fallu rappeler cette originalité de Genève de vouloir lier religion et philosophie moderne, croyance et raison. Soucieuse de préserver le christianisme, elle fut surtout dirigée contre une influence française jugée trop " critique ", " immorale " et sceptique, celle de Voltaire en particulier (un voisin de Genève). Renforcé par les philosophies anglaise et, surtout, écossaise, ce " modèle " a durablement marqué Guizot, qui tient Genève pour son " berceau intellectuel ".

Cette première partie se termine par le retour de Guizot à Paris, en 1805. Pierre-Yves Kirschleger souligne justement l'importance de sa rencontre, en 1807, de Stapfer, un émigré suisse : celui-ci l'initie à la culture allemande, fortifie sa foi (il est pasteur luthérien) et lui montre encore toute l'importance de la morale. Pourtant, à en croire l'auteur, la foi de Guizot se serait éteinte lorsqu'il s'est mis à fréquenter, autour de 1810, la grande société parisienne: " son christianisme a disparu au contact de la philosophie contemporaine" (p.58), et il " ne voit plus dans l'esprit humain que la raison " (p. 63). Affirmations exagérées, et nuancées quelques lignes plus loin (Dieu se serait simplement "voilé" pour Guizot, qui cesse d'aller au temple). Mais ces appréciations auraient du être encore plus nuancées : parce que la pensée de Guizot repose fondamentalement sur la leçon apprise à Genève d'un accord nécessaire entre christianisme et rationalisme, entre croyance et science répondant aux deux aspirations de l'homme. Si la raison est la forme moderne de son intelligence (les leçons de P. Prévost), l'homme n'a pas moins besoin de croire. Aussi ne faut-il pas surestimer l'influence des Idéologues et des héritiers du XVIIIe siècle. Car après Genève, Guizot, même s'il fréquente leurs salons, reste plus proche des milieux spiritualistes. De fait, ces autres rationalistes s'opposent aux Idéologues en s'attachant aussi à unir raison et religion, comme à défendre la primauté de l'éthique contre "l'immoralisme" des Lumières. Il en est ainsi de Stapfer, défenseur du kantisme, qui a beaucoup marqué Guizot, de Royer-Collard (un catholique jansénisant divulgateur de la philosophie écossaise, qui n'est pas signalé)... Chez Guizot, qui demeure chrétien, le problème, en fait, sera de trouver ce difficile équilibre entre foi et raison. Même si le kantisme lui a intellectuellement permis de les concilier, il a évolué, hésitant sur leur part respective. Vers 1810, si la raison l'emporte effectivement dans son esprit ce n'est pas au prix de sa foi (même si elle l'affaiblit).

La seconde partie - les années spiritualistes - envisagent Guizot de la fin de l'Empire à la Révolution de 1848 dans trois domaines, en relation avec sa religion: éducation, histoire et politique. Pierre-Yves Kirschleger montre bien que Guizot est, depuis longtemps, un pédagogue: en 1811 il fonde des Annales de l'Éducation nourries d'expériences étrangères (d'abord suisses et allemandes). Adepte de la pédagogie des Lumières, surtout suisse, il est partisan des méthodes libérales, respectueuses des individus. Leur objectif est de former un homme libre et moral, de futurs citoyens responsables en somme. Mais l'important pour Guizot est le "gouvernement des esprits ": dans une France déstabilisée par la Révolution, il veut, d'abord, moraliser, éduquer le peuple, donc développer son niveau d'enseignement (trop négligé). Cette 
tâche est du ressort de l'État, mais il faut que l'éducation morale, prioritaire, soit aussi religieuse. D'où, en juin 1833, la grande loi Guizot sur l'enseignement primaire : État et Églises, instituteurs et prêtres, doivent collaborer à cette grande oeuvre. P.Y. Kirschleger en montre bien l'enjeu, mais il aurait pu rappeler le précédent de la grande loi sur l'enseignement primaire de Stapfer (décidée, en 1798, dans le cadre de la République-sœur helvétique, dont il était ministre, et qui a influencé Guizot). Mais pourquoi appeler le christianisme à une telle responsabilité dans l'éducation moderne? L'histoire aide à répondre à cette question. L'auteur l'aborde en montrant bien que chez Guizot le christianisme joue un rôle majeur dans l'histoire européenne (thèse développée dans ses cours). En y assurant le développement progressif de l'Humanité il a fondé une " civilisation chrétienne ". Reposant sur la liberté et la responsabilité morales, véritable " révolution morale ", il a d'abord lutté contre le " mal ", notion essentielle chez Guizot qu'il aurait été bon d'approfondir (conséquence du Péché originel ?) Il aurait fallu encore plus souligner l'originalité de son analyse: contre Voltaire et Bossuet, il unit la " civilisation ", héritage des Lumières, au christianisme, religion progressiste. Il est encore redevable à Stapfer, chrétien et homme des Lumières, de cette conception. Dans ces conditions, Guizot ne pouvait que discuter l'anti-christianisme de l'Histoire de la décadence et de la chute de l'Empire romain d'Ed. Gibbon, dont il donne, en 1812, une édition critique. Pierre-Yves Kirschleger note justement la " protestation de sa foi devant l'irréligion de l'historien anglais " (p. 90), mais a tort de penser que sa " perte " de foi aurait pu le conduire, ensuite, à l'approuver (p. 91). D'esprit voltairien, Gibbon dénonce le christianisme pour avoir précipité, avec les Barbares, la chute du grand et humaniste Empire romain. Chute salutaire, riposte Guizot, car en mettant fin à un régime despotique et rétrograde le christianisme a favorisé le progrès, d'abord moral, de l'Europe. De plus, il voit dans l'héroïsme des chrétiens persécutés, mis en doute par Gibbon, la force morale d'une " résistance " (souvenir du Désert?). Autre preuve de la force progressiste du christianisme: la Réforme. Pour Guizot, l'auteur le montre bien, elle a libéré les consciences en proclamant le droit de libre-examen. Mais de religieux le mouvement allait nécessairement gagner l'ordre civil et y favoriser les révolutions (en premier celle d'Angleterre, révolution protestante). Mais ce christianisme ne serait-il qu'utilitariste? Si Guizot, prétend Pierre-Yves Kirschleger, a perdu (?) la foi, Dieu serait-il, dès lors, absent ? En fait non : dans la leçon $\mathrm{V}$ de son cours d'Histoire de la civilisation en Europe (1828) il montre, au contraire, que la raison n'étant pas toute-puissante, l'homme a besoin du secours de Dieu, donc de prier celui dont la "loi supérieure" guide sa marche. Foi " éclairée " sans doute, mais foi tout de même. Guizot reste un chrétien.

Mais il est d'abord protestant: puisque sa communauté a été réintroduite dans la communauté nationale, quelle place y occupe-t-il ? Abordant maintenant le champ politique, P.Y. Kirschleger nous montre bien qu'aux yeux des catholiques il demeure un protestant. Ses interventions pour ses coreligionnaires auprès des autorités comme ses activités dans les sociétés de la Réforme suffiraient à leur donner raison. Pourtant, lorsqu'il exerce le pouvoir, Guizot, l'auteur le souligne justement, ne mène pas de " politique protestante ". Remarque essentielle. De fait notre premier grand dirigeant protestant défend la liberté et la paix religieuses, recherche l'alliance de l'Église catholique (au point de susciter des réactions hostiles chez les siens!). Pierre-Yves Kirschleger en fournit maints exemples. Mais sans nous en donner toutes les raisons. Même si Guizot voit dans la Réforme le christianisme " moderne ", le catholicisme, c'est un fait, est majoritaire et " incontournable " : il faut donc l'intégrer dans la France post- 
révolutionnaire et mettre à son profit, notamment dans l'enseignement, toute sa force morale. Politique approuvée par des catholiques, qui avaient apprécié ses cours, mais, de tous côtés, elle rencontre de fortes résistances.

Au terme de ces " années spiritualistes ", transition vers la troisième partie, Pierre-Yves Kirschleger conclut au retour à la foi de Guizot: " 1811, Dieu s'est voilé. 1830, le voile s'est définitivement déchiré " (p.139). Lui supposant un " credo théiste sommaire et inavoué " (p.141), il étudie précisément cette évolution à travers différents écrits (études et correspondance). Situant de 1828 à 1830 le tournant décisif, il repère aussi les influences de l'entourage: ainsi celles de sa mère, venue définitivement vivre à ses côtés à partir de 1823, et de la duchesse de Broglie, qui est aussi une fervente protestante. Sa participation à des sociétés issues du Réveil aurait aussi pesé. Mais l'auteur voit dans la perte, douloureusement ressentie, de Pauline de Meulan, son épouse, en 1827, la cause décisive de son retour à la foi. Mais que conclure des textes? En 1827 Guizot écrit des Méditations sur l'immortalité de l'âme, puis Quel est le vrai sens du mot foi ? (1828). Comme le montre Pierre-Yves Kirschleger sa religion, de fait, y apparait surtout philosophique. Pourtant l'âme inquiète de Guizot n'aura jamais cessé de s'interroger et, pour notre part, nous ne pensons pas qu'il ait cessé de croire. Tout au plus a-t-il douté. Citons en brièvement quelques preuves : la critique de Gibbon lui a fait prendre conscience de la dimension supra-historique, transcendante, du christianisme (et pourquoi avoir surtout rectifié les passages anti-chrétiens ?); en 1821-1822 il publie De la peine de mort en matière politique, et Des conspirations et de la justice politique, questions graves qu'il place sous l'autorité de la religion ; celle-ci inspire, peu après, ses Méditations dont la première, Sur l'immortalité de l'âme, est précédée d'une prière où il évoque " la foi que je n'ai point perdue " (partie absente de l'édition de 1851). En fait, l'axe de sa pensée aura sans cesse évolué entre croyance et raison. Pierre-Yves Kirschleger se fonde trop sur un article tardif où Guizot juge sévèrement son passé rationaliste (Le christianisme et le spiritualisme, paru en 1869 dans la Revue des DeuxMondes.

La troisième partie - l'apôtre du christianisme, sa pensée religieuse - vient, en quelque sorte, couronner l'ensemble. Soulignant, avec justesse, l'importance de 1848, Pierre-Yves Kirschleger nous montre le repli et le raidissement intellectuels de Guizot. Désormais écarté de la vie publique, contrairement à d'autres anciens responsables de la Monarchie de Juillet, il se consacre essentiellement à des activités intellectuelles. Attribuant au " mal ", qu'il aurait sous-estimé, son échec politique, Guizot va réfléchir aux moyens de combattre les forces et les manifestations de la dissolution sociale. Mais l'homme politique, qu'il reste, apporte des réponses surtout chrétiennes à ses préoccupations. Guizot, qui a rallié les évangéliques du Réveil, devenus majoritaires dans l'Église réformée en 1850, affiche maintenant une foi plus ferme. Il multiplie les écrits religieux, où il se fait penseur chrétien (plus que théologien) : quatre séries de Méditations paraissent de 1851 à 1868, ainsi que L'Église et la société chrétienne en 1861. Toujours favorable au rapprochement entre protestants et catholiques, comme à la coopération des Églises et de l'État (non à leur confusion), il combat résolument pour le triomphe du christianisme: seul il peut apporter un vrai progrès à la France que menacent le "mal" et une nouvelle montée d'irréligion (athéisme, matérialisme, scientisme...). Pierre-Yves Kirschleger observe ainsi que " la défense du christianisme est étroitement liée à la défense de l'idéal politique qu'il a toujours professé " (p. 166). Ses analyses, très précises, des écrits de Guizot montrent qu'il n'a pas fondamentalement évolué : lié à la France moderne, nécessaire à l'accomplissement de 
la liberté et au triomphe de la morale, le christianisme ne s'oppose ni à la science ni à la philosophie. Seulement, l'axe de sa pensée s'est à nouveau déplacé : maintenant, les dogmes, la croyance au surnaturel, y prennent une plus grande place, limitant, dès lors, la part de la raison (sans la contester). Ce christianisme est une religion à la fois régénératrice et régulatrice : elle concerne autant l'âme que la vie sociale. C'est bien ainsi, conclut P.Y. Kirschleger, qu'elle constitue l'une des clés - indispensables - de Guizot. Son ouvrage nous aide, par là, à mieux comprendre la pensée et l'action d'un homme politique et d'un intellectuel qui se voulut plus chrétien, en fait, que protestant. 